\title{
Laparoendoscopic rendezvous may be an effective alternative to a failed preoperative endoscopic retrograde cholangiopancreatography in patients with cholecystocholedocholithiasis
}

\author{
Fotios Tsiopoulos ${ }^{\mathrm{a}}$, Andreas Kapsoritakis ${ }^{\mathrm{a}}$, Athanassios Psychos ${ }^{\mathrm{a}}$, Anastasios Manolakis ${ }^{\mathrm{a}}$, \\ Konstantinos Oikonomou ${ }^{a}$, George Tzovaras ${ }^{b}$, loannis Baloyiannis ${ }^{b}$, Alexandra Tsikrikac, \\ Spyros Potamianos ${ }^{\mathrm{a}}$
}

Larissa University Hospital, Larissa, Greece

\begin{abstract}
Background Endoscopic retrograde cholangiopancreatography (ERCP), followed by laparoscopic cholecystectomy (LC), remains the standard way of management for patients with cholecystocholedocholithiasis. Laparoendoscopic rendezvous (LERV), a combined procedure for removing the gallbladder laparoscopically and clearing the common bile duct (CBD) endoscopically at the same time, could be an attractive alternative. The aim of this study was to compare LERV with classic ERCP in patients with cholecystocholedocholithiasis.

Methods 886 patients with cholecystocholedocholithiasis were treated either with the LERV technique (90 patients), or with the 2-stage approach, which includes preoperative ERCP followed by LC (796 patients). The primary endpoint was any difference in the success of CBD cannulation and clearance; secondary endpoints were the detection of differences in morbidity (especially post-ERCP pancreatitis [PEP]), and the feasibility of the two approaches.

Results Successful cannulation of the CBD was more frequent with conventional ERCP compared with the LERV technique ( $89.8 \%$ vs. $75.5 \%, \mathrm{P}=0.0001)$. LERV appears to be as effective as conventional ERCP for complete CBD clearance (85.5\% vs. $82.8 \%, \mathrm{P}<0.1$ ). None of the patients in the LERV group had an episode of clinical PEP, whereas in the conventional ERCP group there were 23 episodes of PEP and one death. The median amylase level was higher in patients undergoing conventional ERCP group compared to patients in LERV group.
\end{abstract}

Conclusion Classic ERCP has a higher rate of successful CBD cannulation and a similar rate of CBD clearance compared to LERV.

Keywords Laparoendoscopic rendezvous, endoscopic retrograde cholangiopancreatography, cholecystocholedocholithiasis

Ann Gastroenterol 2018; 31 (1): 1-7

Departments of a Gastroenterology (Fotios Tsiopoulos, Andreas Kapsoritakis, Athanassios Psychos, Anastasios Manolakis, Konstantinos Oikonomou, Spyros Potamianos), ${ }^{b}$ Surgery (George Tzovaras, Ioannis Baloyiannis), ${ }^{\mathrm{C}}$ Radiology (Alexandra Tsikrika), Larissa University Hospital, Larissa, Greece

\section{Conflict of Interest: None}

Correspondence to: Fotios Tsiopoulos, 9 Neofytou Street, 41222

Larissa, Greece, e-mail: ftsiopoulos@yahoo.com

Received 2 July 2017; accepted 20 September 2017; published online 2 November 2017

DOI: https://doi.org/10.20524/aog.2017.0210

\section{Introduction}

Endoscopic retrograde cholangiopancreatography (ERCP) has been one of the most common methods for diagnosing and managing choledocholithiasis for several years [1-6]. In fact, it still remains the gold standard method of treatment for choledocholithiasis, despite the fact that magnetic resonance cholangiopancreatography (MRCP) and endoscopic ultrasound (EUS) are also used as the principle diagnostic tools $[7,8]$. Although there is evidence from prospective randomized trials suggesting that the so-called one-stage management 
of cholecystocholedocholithiasis is very effective [9-11], two-stage techniques, mainly preoperative ERCP followed by laparoscopic cholecystectomy (LC), are widely preferred by most clinicians.

However, ERCP may present some disadvantages and complications, one of which is a failure to cannulate the ampulla of Vater, with a rate ranging from 4-18\% [12]. Moreover, post-ERCP pancreatitis (PEP) could result from inadvertent pancreatic cannulation and contrast injection, while general anesthesia may be required when patients are unable to undergo or tolerate ERCP for various reasons. An effective, though technically demanding, method for such cases could be the laparoscopic exploration of the common bile duct (CBD) during the laparoscopic cholecystectomy, which should ideally be equipped with a flexible laparoscopic choledochoscope. Alternatively, another effective procedure could be the so-called laparoendoscopic rendezvous (LERV) $[9,10]$, during which the gallbladder is removed laparoscopically and the CBD is cleared endoscopically, while selective cannulation is simultaneously applied. This method does not require additional equipment apart from what is commonly used in the classic LC and ERCP procedures.

In this paper the gastroenterologist's experience with LERV is presented, focusing on the advantages and disadvantages of this technique, which is directly compared with the established routine approach of ERCP followed by LC.

\section{Patients and methods}

\section{Patients}

Between May 2006 and May 2012 a total of 886 consecutive patients were admitted to the Department of Gastroenterology, University Hospital of Larissa, Greece, with symptomatic cholecystocholedocholithiasis and underwent ERCP and endoscopic sphincterotomy (ES). Among these, 90 patients were treated with the LERV technique and 796 with the standard two-stage approach (ERCP/LC). The LERV group consisted of 56 female and 34 male patients, with a median age of 68 years (range 13-89), while the two-stage group comprised 449 females and 347 males, with a median age of 68.3 years (range 17-97). The age and sex distributions were similar in both groups ( $\mathrm{P}>0.1$ in both cases). Fifty patients of the LERV group were derived from a completed cooperative study of the Departments of Surgery and Gastroenterology at the University Hospital of Larissa, in which the LERV technique had been used [13]. The remaining 40 patients in the same group were either those in which ERCP had failed (31 patients) or patients who refused or were unable to undergo preoperative ERCP as part of the standard two-stage approach (9 patients) (Table 1).

In the University Hospital of Larissa, approximately 900 ERCPs are performed per year, either as emergency or elective cases, all of which involve benign or malignant biliary disease. The combined method of preoperative ERCP/ES with CBD clearance, followed by LC during the same admission, comprises the standard CBD stone management protocol.
Table 1 Demographic and clinical characteristics of patients

\begin{tabular}{lccc} 
Characteristics & LERV & & ERCP \\
\cline { 2 - 2 } & $\mathrm{n}=90$ & & $\mathrm{n}=796$ \\
\hline Sex & 34 & & 347 \\
Male & 56 & & 449 \\
Female & $68(13-89)$ & & $68.3(17-97)$ \\
Age in years (range) & & \\
Clinical characteristics & 21 & 345 \\
Cholangitis & 19 & 163 \\
Pancreatitis & 42 & 177 \\
Elevated LFTs and biliary type & & \\
pain & 8 & 82 \\
Acute cholecystitis & 0 & 4 \\
SOD & 0 & 25 \\
Elevated LFTs & & \\
\hline
\end{tabular}

LERV, laparoendoscopic rendezvous; ERCP, endoscopic retrograde cholangiopancreatography; LFTs, liver function tests; SOD, sphincter of Oddi dysfunction

However, the LERV technique has been alternatively offered to patients with cholecystocholedocholithiasis since May 2006, when this method was adopted in our hospital. In fact, LERV can effectively be used instead of ERCP under benzodiazepine sedation, as part of a two-stage approach, if the patient is unwilling to undergo or is intolerant of the latter method.

MRCP is initially conducted in patients who are at intermediate risk of having cholecystocholedocholithiasis. This group includes patients diagnosed with acute biliary pancreatitis, as well as cases with a moderately dilated bile duct and/or deranged liver function tests. In contrast, MRCP is not routine practice for patients at high risk for cholecystocholedocholithiasis. Patients in this group may suffer from acute cholangitis, obstructive jaundice combined with biliary colic, and CBD stones may have also been traced during the ultrasound (US) scan [14]. For this subgroup of patients, the LERV procedure is commonly followed in both emergency and elective cases.

\section{LERV}

The LERV procedure requires close collaboration and synchronization among surgeons, anesthesiologists and gastroenterologists. The endoscopic team is charged with the transfer and installation of the endoscopic equipment in the operating room.

The LERV procedure, described in detail by Tzovaras et al [13], starts with the laparoscopy, during which the patient is under general anesthesia, in the supine position. More specifically, the cystic duct is dissected free and clipped toward Hartmann's pouch, soon after the dissection of Calot's triangle and the clip ligation of the cystic artery have been performed. 
Following this, intraoperative cholangiography is performed, by inserting a 7-Fr catheter into a small opening just below the clip ligation of the cystic duct, with the purpose of confirming the duct stones initially detected during US and MRCP. C-arm fluoroscopy is used during the whole stage, completed when a jagwire is advanced through the catheter into the duodenum. The next stage involves endoscopy, whereby the wire is first trapped into a polypectomy loop and then pulled through the mouth, with the scope remaining in situ. In this way, one end of the wire comes out of the cholangiography forceps and the other through the mouth. What follows is elective CBD cannulation, conducted by the means of a sphincterotome advanced over the wire, which is removed through the mouth, and remains in the distal CBD. At the same time, the laparoscopist clips and sections the cystic duct. Sphincterotomy is then performed, together with catheter CBD clearance under fluoroscopic guidance. Finally, the endoscope is withdrawn, under simultaneous air suction, and the gallbladder is dissected from the liver bed. A corrugated gravity drain is routinely placed in the subhepatic space.

\section{ERCP}

ERCPs are performed in the radiology suite with the patient in a prone position under benzodiazepine sedation. A triple lumen sphincterotome and the guide wire-assisted technique for $\mathrm{CBD}$ cannulation are used. Then, selective cannulation contrast is injected to opacify the CBD and intrahepatic ducts. If cannulation attempts have not been successful after $10 \mathrm{~min}$, precut sphincterotomy is attempted. ES to facilitate CBD drainage and stone extraction is always performed. Balloon or basket catheters under fluoroscopic guidance are used for stone extraction. Depending on the size of the stones, dilatation and/ or mechanical lithotripsy may also be applied. In the case of incomplete CBD stone removal, a stent is inserted in order to facilitate CBD drainage and a second ERCP for mechanical lithotripsy is attempted within a few weeks.

The patients of both groups were treated by the same personnel in exactly the same way. More specifically, one of the three highly experienced endoscopists, each with a minimum total of 1000 procedures, performed all the endoscopic procedures and the same surgical team participated in all the laparoscopic procedures. The measurements of serum amylase levels were conducted systematically before and $12 \mathrm{~h}$ after the endoscopic procedure. When there were no complications, there was immediate patient mobilization and food intake on the morning following the operation day. The patients were discharged after completing an uneventful in-hospital recovery period of at least $24 \mathrm{~h}$.

\section{Statistical analysis}

The primary endpoint was the success of treatment, estimated on the basis of the following factors: a) the success of the LERV, i.e., the effective cannulation of CBD using the guidewire to reach the duodenum through the cystic duct; b) the success of the ERCP, i.e., the cannulation of $\mathrm{CBD} ; \mathrm{c})$ effective stone extraction, i.e., complete clearance of $\mathrm{CBD}$ stones on the completion cholangiogram; and d) the complication rate, which mainly refers to PEP, i.e., the pancreatic-like pain that persists for at least $24 \mathrm{~h}$ after the procedure associated with serum amylase levels more than three times the upper normal limit. CT was used to confirm pancreatic inflammation.

Categorical variables were compared using the $\chi^{2}$ test with Yates's correction and Fisher's exact test (two-tailed) when necessary. Continuous variables were compared using the Student's $t$-test or the Mann-Whitney $U$ test, depending on distribution. All P-values were two-sided. A P-value of $<0.05$ indicated a statistically significant difference. All calculations were performed using SPSS software (version 10.0) (SPSS Inc., Chicago, IL). Using a web-based power and sample size calculator it was estimated that at least 60 and 530 subjects, respectively, had to be included in the LERV and ERCP treatment arms in order to achieve a power of $80 \%$ at the 0.05 level of significance.

\section{Ethical considerations}

The study was approved by the University of Thessaly Medical School's Ethics Committee. Informed consent was obtained from all study patients.

\section{Results}

\section{LERV-related outcomes}

The initial concept of LERV was not achieved (i.e., the cannulation of the CBD with the LERV technique) in 22 of 90 patients (success rate $75.5 \%$ ), because the guidewire introduced via the cystic duct could not be advanced into the duodenum in order to be found, trapped, and pulled out by the endoscopist (Table 2).

In these 22 patients for whom the LERV failed, the CBD was successfully cannulated via the classic endoscopic route, as performed in a routine ERCP, in 19 patients. In one patient we did not proceed to ERCP because of a negative intraoperative

Table 2 Causes of failure to advance the guidewire into the abdomen

\begin{tabular}{l} 
- Cystic duct abnormality or stone in 13 cases \\
- CBD stone in 4 cases \\
- CBD stricture in 1 case \\
- Juxta-papillary diverticulum in 1 case \\
- Ampullary mass in 1 case \\
- Dissection of the cystic duct in 1 case \\
- Inability to recognize CBD due to adhesions in 1 case \\
\hline$C B D$, common bile duct
\end{tabular}


cholangiogram, while in two patients the procedure was converted to open (Fig. 1).

Regarding procedure-related complications, no episodes of clinical post-ERCP pancreatitis were recorded. The median amylase value measured $12 \mathrm{~h}$ after the procedure was $65 \mathrm{U} / \mathrm{mL}$ (range 16-1159). Two deaths were recorded, one due to intraabdominal abscess and one due to perforation during ES (1.1\%). Three patients exhibited post-ERCP cholangitis (3.3\%), successfully treated with antibiotics; one patient presented pulmonary embolism (1.1\%) and another one sphincterotomyrelated bleeding (1.1\%), which ultimately required endoscopic intervention.

The simultaneous procedure of $\mathrm{CBD}$ clearing and gallbladder removal was successfully conducted in 77 patients, thus leading to a high success rate (85.5\%). In all cases, CBD clearance was facilitated by ES, which enabled the extraction of a median number of 2 stones (range: 1-5), with a median size of $5 \mathrm{~mm}$ (range: 2-12). Fluoroscopic images were used as means of calculating stone size.

In 13 cases, a remaining large stone prevented complete CBD clearance, even though selective CBD cannulation had been achieved and sphincterotomy performed. Since the operative time was prolonged, a stent was used to facilitate CBD drainage. Mechanical lithotripsy by the means of repeated ERCP was successfully conducted a few weeks later.

Regarding completion time calculations, the entire LERV procedure lasted for a median time of 95 (range 65-200) min, while the respective median time for the endoscopic part was 32 (range 15-70) $\mathrm{min}$.

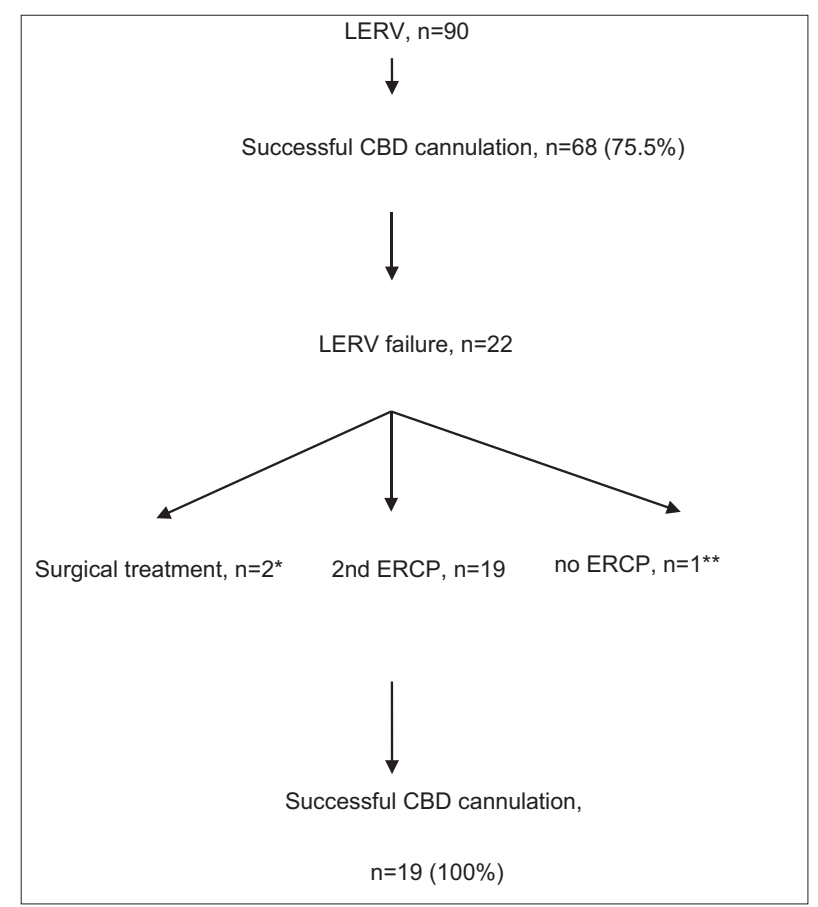

Figure 1 Flow chart

${ }^{*}$ procedure was converted to open: adhesions, cystic duct dissection

${ }^{* *}$ negative intra-operative cholangiogram

\section{ERCP/LC-related outcomes}

During the same period, 796 ERCPs were performed for patients with symptomatic cholecystocholedocholithiasis. Cannulation of CBD and ES were achieved in 715 patients (success rate 89.8\%). Among the 81 patients for whom ERCP failed, 72 patients presented a difficult anatomy (mainly a large diverticulum), which prevented the Vater ampulla cannulation. In addition, three cases of poor patient cooperation caused the premature discontinuation of the procedure. In the other six patients the ampulla of Vater could not be reached because of chronic duodenal ulcer disease in three patients, infiltration of the duodenal bulb due to pancreatic cancer in one patient, previous Billroth II surgery in another patient, and stenosis preventing insertion of the scope into the esophagus in another patient.

ES was performed in all patients in order to facilitate CBD clearance with balloon or basket stone extraction catheters. Depending on the size of the stone, mechanical lithotripsy and dilatation were performed at the site of sphincterotomy. Complete stone removal of a median stone size of $8 \mathrm{~mm}$ (range: sludge to $>25 \mathrm{~mm}$ ) was achieved in 592 cases (success $82.8 \%$ ). In 123 patients complete stone removal could not be achieved for various reasons (Table 3), mainly because of large stone size (>15 mm).

Regarding the complications observed (Table 4), the incidence of post-ERCP pancreatitis was $2.91 \%$ (23 episodes). Severe pancreatitis was documented in three patients $(0.38 \%)$. The medium amylase value measured $12 \mathrm{~h}$ after the procedure was $133.64 \mathrm{U} / \mathrm{mL}$ (range 19-2300). One death due to pancreatitis and prolonged ICU hospitalization was recorded. Seven patients suffered from post-ERCP cholangitis (1.2\%) and were treated with antibiotics. Perforation occurrence was $0.27 \%$ (two patients) and both patients were treated conservatively. One patient exhibited cardiac arrhythmia during the procedure $(0.13 \%)$, resulting in premature discontinuation of the ERCP. Sphincterotomy-related bleeding occurred in two cases $(0.27 \%)$ and was successfully managed by means of endoscopic intervention.

Regarding time duration calculations for the group of patients treated with the two-stage approach, the median ERCP duration was 29 (range 15-60) min, while the median duration for the subsequent LC was higher, reaching 50 (range 25-120) $\min$.

\section{Discussion}

The results of the present study suggest that LERV could be a safe alternative for patients with symptomatic cholecystocholedocholithiasis in whom ERCP as part of a twostage approach has either failed or is considered unsuitable.

The anatomy of the periampullary region and the endoscopist's experience seem to influence CBD cannulation failure during ERCP, reported to range between $4 \%$ and $18 \%$ [14]. Moreover, the study confirms that CBD cannulation 
Table 3 Causes of incomplete CBD clearance

\begin{tabular}{l}
\hline - Large stone (57 cases) \\
- Hemorrhage during sphincterotomy (10 cases) \\
- Poor cooperation of patient (11 cases) \\
- CBD dilanosis (9 cases) \\
- Critical illness (8 cases) \\
- Malignancy ( 2 cases) \\
- Foreign body (stent, basket catheter) in CBD ( 3 cases) \\
- Prolonged procedure (8 cases) \\
- Cardiac arrhythmia (1 case) \\
- Inadequate opacification of CBD (9 cases) \\
-Problem with C-arm fluoroscopy (1 case) \\
\hline CBD, common bile duct
\end{tabular}

Table 4 Complications related to ERCP

\begin{tabular}{lcc}
\hline Complications & ERCP (796 patients) & LERV (90 patients) \\
\hline Pancreatitis & $23(2.91 \%)$ & 0 \\
Cholangitis & $7(1.2 \%)$ & $3(3.3 \%)$ \\
Bleeding from & $2(0.27 \%)$ & $1(1.1 \%)$ \\
ES & & \\
Perforation & $2(0.27 \%)$ & $1(1.1 \%)$ \\
Cardiovascular & $1(0.13 \%)$ & $1(1.1 \%)$ \\
Deaths & $1(0.13 \%)$ & $2(2.2 \%)$ \\
\hline
\end{tabular}

ERCP, endoscopic retrograde cholangiopancreatography;

$L E R V$, laparoendoscopic rendezvous; ES, endoscopic sphincterotomy

is most likely to be impeded by a very common anatomical difficulty, such as a juxta-papillary diverticulum [13], which most of the patients in the sample presented.

However, ERCP entails various and serious complications, one of which is acute pancreatitis. In fact, the incidence of PEP usually ranges from 5-10\%, although it may exceed $25 \%$ in certain high-risk populations [15]. In the University Hospital of Larissa, where the present research was conducted, the common practice is for ERCP procedures to be performed in the radiology suite, where the patient is under sedation but without anesthesia or even anesthetic supervision. Therefore, the time for cannulating a "difficult" papilla in low-compliance patients is usually limited [14]. All three endoscopists performing ERCP are considered to be among the most highly experienced, each having participated in at least 1000 procedures. In our study there was a higher cannulation success rate with the classic ERCP compared with the LERV technique $(89.8 \%$ vs. $75.5 \%, \mathrm{P}=0.0001)$. Interestingly, ERCP performed intraoperatively was always successful, even in cases where LERV was unsuccessful. A possible explanation could be that the procedures were performed under anesthesia.

Wang et al [16] enrolled randomized controlled trials (RCTs) in their meta-analysis that compared preoperative and intraoperative ES in patients with gallbladder and suspected
CBD stones. They reported significantly higher failure rates for CBD cannulation in the preoperative ES group, but they referred to studies that did not use the same LERV or ERCP technique and did not define the success of the LERV technique in the same way. Additionally, they did not include patients with a surgically altered anatomy.

Morino et al [9] showed that the LERV technique presented a lower failure rate compared with the sequential ERCP/LC: $5 \%$ vs. $20 \%$. In our opinion, a failure rate of $20 \%$ is extremely high for a high-volume tertiary hospital. This significant difference may be explained in two ways. In difficult cases, the endoscopist avoided taking risks in preoperative ERCP, since he was aware of the fact that CBD cannulation could also be performed during the LC. Moreover, when LERV is conducted in difficult patients, the cannulation of the papilla can be facilitated by the use of a guidewire, passing through the cystic duct into the CBD to reach the duodenum.

The main reason for LERV failure was the inability to introduce the guidewire through the cystic duct. El Geidie et al [17] found the same results in their controlled randomized study. In particular, they tried to pass a guided wire through the cystic duct into the CBD to facilitate bile duct cannulation at the subsequent endoscopy (the endolaparoscopic "rendezvous" described by Cavina et al), but found it technically difficult and omitted this step in most cases.

In fact, LERV seems to be as effective as classic ERCP for complete CBD clearance $(85.5 \%$ vs. $82.8 \%, \mathrm{P}>0.1)$, although the stones extracted during LERV are usually smaller (median diameter $5 \mathrm{~mm}$ vs. $8 \mathrm{~mm}$ ). Complete CBD clearance of large stones using sphincterotomy dilatation and mechanical lithotripsy in the operating room would be time consuming and would prolong the time of surgery.

One of the main advantages of the rendezvous approach is the elective CBD cannulation, because it seems to reduce the possibility of post-procedural pancreatitis, as was clearly demonstrated in two relevant RCTs $[10,14]$. In our study none of the patients in the LERV group had an episode of clinical PEP, while in the classical ERCP group 23 episodes of PEP and a death occurred. The median value of amylase, measured $12 \mathrm{~h}$ after the procedure, was higher in patients in the classic ERCP group than in patients in the LERV group (133.64 vs. 65, $\mathrm{P}<0.001)$. A value of amylase three times lower than normal, after the procedure, has almost never been associated with clinical pancreatitis. However, it has been speculated that hyperamylasemia represents a sign of pancreatic damage, which is not always clinically interpreted as an episode of acute pancreatitis. The fact that the LERV group had a lower incidence of hyperamylasemia might suggest that LERV is safer than standard ERCP as far as the possibility of PEP is concerned, although it rarely occurs. The occasionally increased postprocedure amylase value may be due to the manipulation of the sphincter during sphincterotomy and stone extraction.

Another advantage of the LERV technique over traditional ERCP concerns the method of contrast injection through the cystic duct. In particular, in the LERV technique, one of the first stages is the so-called "intraoperative cholangiogram", performed with contrast injection through the cystic duct, in contrast to the retrograde injection, typically performed in 
traditional ERCP. There is evidence that, if contrast material is accidentally introduced into the pancreatic duct under high pressure, it may cause post-ERCP pancreatitis [18]. Interestingly, in our study there was an inadvertent contrast injection into the pancreatic duct because of a stone that had been impacted in the distal CBD, but the patient did not experience an acute pancreatitis. In the case of inadvertent pancreatic duct cannulation and/or contrast injection, several studies have demonstrated that the use of a short, 3 Fr or 5 Fr pancreatic stent could minimize the risk of mild or moderate PEP, although the risk of severe post-ERCP pancreatitis does not seem to be eliminated [18].

Regarding the postoperative treatment, although previous studies reported a reduction in hospital stay by an average of 1.5 days in the LERV group [13], in our study we could not make this comparison, since many patients who underwent an uneventful ERCP had to prolong their admission because of various reasons irrelevant to the ERCP.

Regarding the technical problems of the combined approach, most relate either to the supine position, which may cause difficulties in the retrograde cannulation of the papilla, or to the gas needed for endoscopy, which may possibly interfere with LC because of the distention of bowel loops. In fact, El Geidie et al [17] faced problems with the cannulation of the papilla in the supine position, thus having to turn the patient to the prone position to complete ERCP.

Although there is evidence from several studies that the LERV technique is effective, it has gained less popularity than expected. This may be due to organizational, logistical and technical problems, such as the availability and training of surgical and endoscopic teams and the prolonged use of the operating room. However, in our hospital successful coordination between the surgical and the endoscopic teams was achieved, since there was availability on a daily basis. It was also obvious that the technical facilities available in the Radiology Department were much better than those in the operating room. Consequently, our equipment had to be transferred and re-installed. As regards the endoscopic team, the time required for the classical ERCP was much shorter than the time for the LERV technique.

It is difficult to perform the LERV technique in critically ill patients. Although in a previous study Tzovaras et al [19] included emergency cases, in most of the RCTs patients with acute cholangitis or necrotizing biliary pancreatitis were excluded $[9,10,17]$. According to the literature, early ERCP is recommended for patients with acute cholangitis superimposed on acute pancreatitis [20]. In our department, ERCP is performed in patients with septic cholangitis or severe biliary pancreatitis within $24 \mathrm{~h}$ from their admission.

Our study is a retrospective, non-randomized controlled study that reflects the daily clinical practice in a high volume, tertiary referral university hospital. Our aim was to extend and complete previously published studies from our hospital, focusing on the gastroenterological aspects of the whole process $[13,19]$. Another limitation of this study is the imbalance in the number of patients who underwent ERCP/ LC and LERV.

In conclusion, provided that there is expertise and advanced

\section{Summary Box}

\section{What is already known:}

- Endoscopic retrograde cholangiopancreatography (ERCP) has been the gold standard for the diagnosis and management of choledocholithiasis

- Despite the evidence from prospective randomized trials suggesting the superiority of the so-called one-stage management of cholecystocholedocholithiasis (LERV), two-stage techniques, mainly preoperative ERCP followed by laparoscopic cholecystectomy, are currently being used by most clinicians

- ERCP is associated with a rate of failure to cannulate the ampulla of Vater ranging from $4-18 \%$

- Post-ERCP pancreatitis (PEP) is one of the concerns in patients who undergo ERCP

\section{What the new findings are:}

- ERCP has higher rate of successful common bile duct (CBD) cannulation compared to LERV

- ERCP has a similar rate of CBD clearance compared to LERV

- Patients undergoing LERV have a lower incidence of PEP and hyperamylasemia

equipment, the LERV procedure could be a safe and attractive alternative for the management of concomitant cholelithiasis and choledocholithiasis, particularly in cases with patientrelated risk factors for acute post-ERCP pancreatitis or in cases in which ERCP has already failed to solve the problem. Although repeat ERCP under anesthesia seems to have higher success rates than LERV, LERV remains the most reasonable alternative in cases where cholecystectomy cannot be further delayed and is therefore going to be performed as a first procedure.

\section{Acknowledgment}

The authors would like to thank the statisticians of the Anavasis group for statistical analysis.

\section{References}

1. Vaira D, D’Anna L, Ainley C, et al. Endoscopic sphincterotomy in 1000 consecutive patients. Lancet 1989;2:431-434.

2. Scientific Committee of the European Association for Endoscopic Surgery (E.A.E.S.). Diagnosis and treatment of common bile duct stones (CBDS). Results of a consensus development conference. 
Surg Endosc 1998;12:856-864.

3. Carr-Locke DL. Therapeutic role of ERCP in the management of suspected common bile duct stones. Gastrointest Endosc 2002;56(6 Suppl):S170-S174.

4. Baillie J, Paulson EK, Vitellas KM. Biliary imaging: a review. Gastroenterology 2003;124:1686-1699.

5. Ausch $\mathrm{C}$, Hochwarter $\mathrm{G}$, Taher $\mathrm{M}$, et al. Improving the safety of laparoscopic cholecystectomy: the routine use of preoperative magnetic resonance cholangiography. Surg Endosc 2005; 19:574-580.

6. Cuschieri A, Lezoche E, Morino M, et al. E.A.E.S. multicenter prospective randomized trial comparing two-stage vs single-stage management of patients with gallstone disease and ductal calculi. Surg Endosc 1999;13:952-957.

7. Rhodes M, Sussman L, Cohen L, Lewis MP. Randomised trial of laparoscopic exploration of common bile duct versus postoperative endoscopic retrograde cholangiography for common bile duct stones. Lancet 1998;351:159-161.

8. Enochsson L, Lindberg B, Swahn F, Arnelo U. Intraoperative endoscopic retrograde cholangiopancreatography (ERCP) to remove common bile duct stones during routine laparoscopic cholecystectomy does not prolong hospitalization: a 2-year experience. Surg Endosc 2004;18:367-371.

9. Morino M, Baracchi F, Miglietta C, Furlan N, Ragona R, Garbarini A. Preoperative endoscopic sphincterotomy versus laparoendoscopic rendezvous in patients with gallbladder and bile duct stones. Ann Surg 2006;244:889-893.

10. Lella F, Bagnolo F, Rebuffat C, Scalambra M, Bonassi U, Colombo E. Use of the laparoscopic-endoscopic approach, the socalled "rendezvous" technique, in cholecystocholedocholithiasis. A valid method in cases with patient-related risk factors for postERCP pancreatitis. Surg Endosc 2002;20:419-423.

11. Rajnakova A, Goh PM, Ngoi SS, Lim SG. ERCP in patients with periampullary diverticulum. Hepatogastroenterology 2003;50:625-628

12. Freeman ML, DiSario JA, Nelson DB, et al. Risk factors for postERCP pancreatitis: a prospective, multicenter study. Gastrointest Endosc 2001;54:425-434.

13. Tzovaras G, Baloyiannis I, Kapsoritakis A, Psychos A, Paroutoglou G, Potamianos S. Laparoendoscopic rendezvous: an effective alternative to a failed preoperative ERCP in patients with cholecystocholedocholithiasis. Surg Endosc 2010;24:2603-2606.

14. Targarona EM, Bendahan GE. Management of common bile duct stones: controversies and future perspectives. HPB (Oxford) 2004;6:140-143.

15. Freeman ML, Guda NM. Prevention of post-ERCP pancreatitis: a comprehensive review. Gastrointest Endosc 2004;59:845-864.

16. Wang B, Guo Z, Liu Z, et al. Preoperative versus intraoperative endoscopic sphincterotomy in patients with gallbladder and suspected common bile duct stones: system review and metaanalysis. Surg Endosc 2013;27:2454-2465.

17. ElGeidie AA, ElEbidy GK, Naeem YM. Preoperative versus intraoperative endoscopic sphincterotomy for management of common bile duct stones. Surg Endosc 2011;25:1230-1237.

18. Choudhary A, Bechtold ML, Arif M, et al. Pancreatic stents for prophylaxis against post-ERCP pancreatitis: a meta-analysis and systematic review. Gastrointest Endosc 2001;73:275-282.

19. Tzovaras G, Baloyiannis I, Zachari E, et al. Laparoendoscopic rendezvous versus preoperative ERCP and laparoscopic cholecystectomy for the management of cholecystocholedocholithiasis: interim analysis of a controlled randomized trial. Ann Surg 2012;255:435-439.

20. Tenner S, Baillie J, DeWitt J, Vege SS; American College of Gastroenterology. American College of Gastroenterology guideline: management of acute pancreatitis. Am J Gastroenterol 2013;108:1400-1415; 1416. 